\title{
Fracturas Maxilofaciales en Individuos Chilenos
}

\author{
Maxillofacial Fractures in Chilean Subjects \\ María Jesús Medina; Paola Molina; Lila Bobadilla; Rodrigo Zaror \& Sergio Olate
}

MEDINA, M. J.; MOLINA, P.; BOBADILLA, L. ZAROR, R. \& OLATE, S. Fracturas maxilofaciales en individuos chilenos. Int. J. Morphol., 24(3):423-428, 2006.

RESUMEN: Las fracturas orales y maxilofaciales representan un 6\% a $8 \%$ de las fracturas óseas corporales. Estas lesiones son de complejo manejo y exigen un diagnóstico precoz, certero y preciso. El Hospital Regional Hernán Henríquez Aravena, de la ciudad de Temuco, no posee cirujanos orales y maxilofaciales en los equipos de urgencia inmediata, de forma que los ingresos son realizados por otros profesionales.

En un año de seguimiento se realizaron 34.931 consultas medico-legales en el Servicio de Urgencia del Hospital Regional Hernán Henríquez Aravena. De esas consultas se escogió una muestra representativa de 742 sujetos de quienes se obtuvo información general y de las lesiones en particular. Se obtuvo también asociación estadística mediante la prueba Chi - Cuadrado.

Seis porciento de las consultas en el Hospital Regional Hernán Henríquez Aravena fueron asociadas a fracturas maxilofaciales, donde el grupo con más consultas fueron el de los menores de 20 años. Del total muestral, el 64.4\% correspondió a sujetos del sexo masculino, manteniendo una relación hombre:mujer de 3.1:1; sin embargo, esta relación aumento su diferencia en sujetos mayores de 15 años. El $31.1 \%$ de las consultas fueron causadas por agresiones, mientras que $24.4 \%$ fueron asociadas a las caídas. La principal zona de fractura fue el complejo orbito-naso-etmoidal con un $55.6 \%$ y las fracturas dentoalveolares con un $22.2 \%$. Existió asociación estadísticamente significativa entre edad, sexo, agresión, fracturas mandibulares y fracturas dentoalveolares $(\mathrm{p}<0.05)$.

Los datos reflejan la alta prevalencia de fracturas maxilofaciales en la comuna de Temuco, Chile, siendo similar a lo que ocurre en otras partes del mundo. El grupo de los jóvenes es el de principal consulta donde las agresiones juegan un papel fundamental. Con el presente estudio descriptivo creemos que se justifica plenamente la presencia de cirujanos orales y maxilofaciales en los equipos de urgencia del Hospital Regional Hernán Henríquez Aravena, Temuco, Chile.

PALABRAS CLAVE: Fracturas maxilofaciales; Trauma maxilofacial; Epidemiología.

\section{INTRODUCCIÓN}

Las lesiones orales y maxilofaciales son de relativa frecuencia en el mundo y, a diferencia de otras lesiones del organismo, son complejas tanto por la estética que representan como también por la complejidad anatómica de la zona (Ellis, 2003).

Las fracturas maxilofaciales exigen un diagnóstico certero, preciso y oportuno ya que la variabilidad del tratamiento dependerá de la edad del paciente, tipo de fractura y complicaciones asociadas, entre otras. De este modo, el trabajo de personal especializado en el área de traumatología oral y maxilofacial es altamente necesario (Cunningham \& Hang, 2004).
El manejo del traumatismo maxilofacial es aun controversial en muchos de sus aspectos (Gerbino et al., 2000), de modo que solo la formación continua de especialistas ayudara al control y manejo de estos pacientes.

El Hospital Regional Hernán Henríquez Aravena de la comuna de Temuco, Chile, es el centro público de salud de mayor complejidad en la IX Región de Chile. De el dependen casi 1.000.000 de personas que habitan la ciudad de Temuco y sus alrededores, en un territorio de 31.842 $\mathrm{km}^{2}$. (Instituto Nacional de Estadística, 2003). En este centro hospitalario existe una unidad de urgencia médica atendida por médicos de urgencia, pediatras, cirujanos del adul- 
to y del niño, pero no existe odontólogo de urgencia nocturna y menos cirujanos orales y maxilofaciales. No obstante, existe un departamento de cirugía y traumatología oral y maxilofacial diurno que atiende derivaciones del servicio de urgencia y de hospitales de menor complejidad.

Planteamos un estudio descriptivo para cumplir con los objetivos trazados que fueron: (a) conocer la prevalencia de fracturas orales y maxilofaciales en el Hospital Regional Hernán Henríquez Aravena de Temuco, Chile, (b) identificar las principales causas de fracturas maxilofaciales y establecer alguna relación ente causa de fractura, zona de fractura y factores epidemiológicos e (c) identificar la real necesidad de contar con un servicio de urgencia que cuente con personal especializado en el área.

\section{MATERIAL Y MÉTODO}

Se condujo un estudio de tipo descriptivo en el Servicio de Urgencia del Hospital Regional Hernán Henríquez Aravena (SUHR). Para el estudio se realizó un seguimiento entre Abril del año 2002 y Marzo del año 2003 en el Servicio de Urgencia de este centro asistencial.

Los criterios de ingreso al estudio fueron: 1. Acudir personalmente al SUHR (consulta primaria o derivación desde un centro de menor complejidad) y 2. Sujeto clasificado con compromiso "médico - legal" (lo que implica registro policial).

De esta forma, el universo del estudio fueron los pacientes ingresados en la fecha mencionada, correspondiendo a 34.931 sujetos. De igual manera, la muestra representativa y aleatoria se conformó de 742 pacientes de ambos sexos y de todas las edades. Todos los ingresos seleccionados se integraron al estudio, incluyendo aquellos que presentasen registros incompletos ya que este punto también seria evaluado. En la Tabla I, que muestra la distribución de la muestra según edad, se aprecia que la mayor cantidad de consulta de urgencia fueron realizadas por hombres y por sujetos de entre 0 y 19 años de edad.

La recepción de los pacientes fue realizada por el equipo de urgencia del mismo hospital quienes realizaron posteriormente las derivaciones al Servicio de Cirugía Oral y Maxilofacial del mismo centro asistencial. Las mediciones realizadas incluyeron elementos como el tipo de accidente y las estructuras óseas maxilofaciales comprometidas en las lesiones.
El análisis de los datos se realizó con un software estadístico que incluyo las prueba Chi-Square, estableciendo para ello el mayor cruce de variables posibles. La significancia estadística se presento cuando $\mathrm{p}<0.05$.

\section{RESULTADOS}

Un total de 45 sujetos (6.1\% de la muestra) presentaron fracturas maxilofaciales; la Tabla II muestra la distribución de tales pacientes según su edad. En el grupo menor de 14 años existió menos demanda por este tipo de lesiones que en los sujetos mayores de 15 años. En cuanto al sexo, (Tabla II) los hombres siempre sufrieron mayor cantidad de fracturas que las mujeres, estableciendo una relación hombre:mujer de 3.1:1; esta relación disminuye en el grupo de los menores de 14 años (1.8:1) y evidentemente aumenta en el grupo de los mayores de 15 años, correspondiendo un $82 \%$ de las fracturados al sexo masculino.

La distribución de la etiología de las fracturas es vista en la Tabla III. En el grupo menor de 14 años la principal causa de fracturas fueron las caídas, quedando los accidentes escolares en segundo lugar. En el grupo mayor de 15 años, la principal causa de fracturas fueron las agresiones y violencia. Para toda la muestra, las agresiones fueron la principal causa de fractura (31\%), seguida de las caídas $(24 \%)$ y los accidentes de tránsito $(11 \%)$.

Las estructuras óseas comprometidas en las fracturas maxilofaciales se aprecian en la Tabla IV. En el grupo de los menores de 14 años, las estructuras más comprometidas fueron la región órbito - naso - etmoidal (ONE) con un 47\% y las estructuras dentoalveolares con igual porcentaje. En el grupo de los mayores de 15 años, el $61 \%$ presentó fracturas de la región ONE, seguido de un $21 \%$ con fractura de mandíbula y un $7.1 \%$ con fractura de hueso cigomático. Finalmente, lo más prevalente en la muestra fueron las fracturas de la región ONE (55.6\%), las fracturas dentoalveolares $(22.2 \%)$ y las fracturas de mandíbula (13.3\%).

El análisis estadístico realizado con la prueba ChiCuadrado se observa en la Tabla V. Luego del análisis de las variables del estudio (etiología de la fractura, estructuras anatómica afectadas, sexo y edad), se obtuvo significancia estadística solo en una parte de ellas. En la tabla V se aprecia que la edad y el sexo son factores relevantes en estas lesiones; de este modo, la primera de ellas se relaciona significativamente con algunos tipos de fractura (de mandíbula y dentoalveolar) y con algunos factores etiológicos, mientras que la segunda se relaciona significativamente con las agresiones y los accidentes escolares. 


\begin{tabular}{ccccc}
\hline & $\begin{array}{c}\text { Muestra de } 742 \\
\text { sujetos } \\
\text { ingresados al } \\
\text { SUHR }\end{array}$ & $\begin{array}{c}\text { Pacientes con presencia } \\
\text { de fractura oral y/o } \\
\text { maxilofacial ingresados } \\
\text { al SURH }\end{array}$ \\
\cline { 2 - 5 } Años & $\mathrm{n}$ & $\%$ & $\mathrm{n}$ & $\%$ \\
$0-5$ & 127 & 17,1 & 6 & 13,3 \\
$6-9$ & 111 & 14,9 & 7 & 15,6 \\
$10-14$ & 130 & 17,5 & 4 & 8,9 \\
$15-19$ & 58 & 7,9 & 4 & 8,9 \\
$20-39$ & 181 & 24,4 & 17 & 37,8 \\
$40-64$ & 93 & 12,5 & 5 & 11,1 \\
65 y más & 42 & 5,7 & 2 & 4,4 \\
Total & 742 & 100,0 & 45 & 100,0 \\
\hline
\end{tabular}

Tabla I. Distribución y porcentaje de la muestra de 742 sujetos y de 45 sujetos con fractura oral y/o maxilofacial, según edad, ingresados entre Abril del año 2002 hasta Marzo del año 2003 al Servicio de Urgencia del Hospital Regional Hernán Henríquez Aravena de Temuco, Chile.

Tabla II. Porcentaje de la muestra de 742 sujetos y de los 45 sujetos con fractura oral y/o maxilofacial, según sexo, ingresados entre Abril del año 2002 hasta Marzo del año 2003 al Servicio de Urgencia del Hospital Regional Hernán Henríquez Aravena de Temuco, Chile.

\begin{tabular}{|c|c|c|c|c|}
\hline \multirow[b]{2}{*}{ Años } & \multicolumn{2}{|c|}{$\begin{array}{c}\text { Muestra de } 742 \text { sujetos ingresados al } \\
\text { SUHR }\end{array}$} & \multicolumn{2}{|c|}{$\begin{array}{c}\text { Pacientes con presencia de fractura oral y/o } \\
\text { maxilofacial ingresados al SURH }\end{array}$} \\
\hline & Femenino & Masculino & Femenino & Masculino \\
\hline $0-14$ & 40,8 & 30,5 & 35,3 & 17,9 \\
\hline 15 y más & 59,2 & 69,5 & 64,7 & 82,1 \\
\hline Total & 100,0 & 100,0 & 100,0 & 100,0 \\
\hline
\end{tabular}

Tabla III. Etiología de las fracturas maxilofaciales según la edad de los 45 individuos con fracturas maxilofaciales ingresados entre Abril del año 2002 hasta marzo del año 2003 al sujetos Servicio de Urgencia del Hospital Regional Hernán Henríquez Aravena de Temuco, Chile.

\begin{tabular}{lcrrrrr}
\hline \multicolumn{1}{c}{ Etiología } & \multicolumn{2}{c}{$0-14$ años } & \multicolumn{2}{c}{15 y más años } & \multicolumn{2}{c}{ Total } \\
\hline \multirow{2}{*}{ Accidentes de tránsito } & $n$ & $\%$ & $n$ & $\%$ & $n$ & $\%$ \\
Agresiones & 1 & 5,9 & 4 & 14,3 & 5 & 11,1 \\
Deportes & 1 & 5,9 & 13 & 46,4 & 14 & 31,1 \\
Caídas & 1 & 5,9 & 2 & 7,1 & 3 & 6,7 \\
Accidentes de trabajo & 9 & 52,9 & 2 & 7,1 & 11 & 24,4 \\
Accidentes escolares & 0 & 0,0 & 0 & 0,0 & 0 & 0,0 \\
No consignado & 4 & 23,5 & 0 & 0,0 & 4 & 8,9 \\
\multicolumn{1}{c}{ Total } & 1 & 5,9 & 7 & 25,0 & 8 & 17,8 \\
\hline
\end{tabular}

Tabla IV. Tipo de estructuras ósea comprometidas en las fracturas maxilofaciales según la edad de la muestra de 45 individuos con fracturas maxilofaciales ingresados entre Abril del año 2002 hasta marzo del año 2003 al sujetos Servicio de Urgencia del Hospital Regional Hernán Henríquez Aravena de Temuco, Chile.

\begin{tabular}{lcccccc}
\hline \multicolumn{1}{c}{ Estructura } & \multicolumn{2}{c}{$0-14$} & \multicolumn{2}{c}{ 15 y más } & \multicolumn{2}{c}{ Total } \\
\hline \multirow{2}{*}{ Mandíbula } & $n$ & $\%$ & $n$ & $\%$ & $n$ & $\%$ \\
Cigomático & 0 & 0 & 6 & 21,4 & 6 & 13,3 \\
Maxila & 0 & 0 & 2 & 7,1 & 2 & 4,4 \\
Orbito- Naso - Etmoidal & 0 & 0 & 0 & 0 & 0 & 0 \\
Frontal & 8 & 47,1 & 17 & 60,7 & 25 & 55,6 \\
Dentoal veolar & 1 & 5,9 & 1 & 3,6 & 2 & 4,4 \\
\multicolumn{1}{c}{ Total } & 8 & 47,1 & 2 & 7,1 & 10 & 22,2 \\
\hline
\end{tabular}


Tabla V. Resultados estadísticamente significativos en la asociación de las variables de estudio mediante prueba estadística Chi-Cuadrado, correspondiente a los 45 individuos con fracturas maxilofaciales ingresados entre Abril del año 2002 hasta marzo del año 2003 al Servicio de Urgencia del Hospital Regional Hernán Henríquez Aravena de Temuco, Chile.

\begin{tabular}{ccc}
\hline Variable 1 & Variable 2 & $p=$ \\
\hline Edad & Fractura mandíbula & 0.0403 \\
Edad & Fractura dentoalveolar & 0.0018 \\
Edad & Agresión & 0.0044 \\
Edad & Accidente escolar & 0.0072 \\
Edad & Caídas & 0.0005 \\
& & \\
Sexo & Agresión & 0.0305 \\
Sexo & Accidente escolar & 0.0330 \\
Sexo & No consignado & 0.0207 \\
Sexo & Fractura frontal & 0.0232 \\
& & \\
Accidente escolar & Fractura dentoalveolar & 0.0078 \\
Agresión & Fractura mandíbula & 0.0433 \\
\hline
\end{tabular}

\section{DISCUSIÓN}

Las fracturas maxilofaciales exigen una constante capacitación del cirujano oral y maxilofacial a fin de optimizar el tratamiento y lograr buenos resultados en el manejo de estas lesiones (Cunningham \& Hang) con el objetivo de priorizar y protocolizar los mejores procedimientos terapéuticos. De hecho, ya Bater et al. (2005) demostraron la variabilidad que existe entre cirujanos maxilofaciales para el manejo de hemorragia retrobulbar asociada a fracturas maxilofaciales.

En el SUHR existe una alta consulta por urgencia general. Siendo la muestra aleatoria un reflejo del universo de estudio, podemos señalar que de las 34.931 consultas, el $6.1 \%$ corresponde a urgencias por fracturas maxilofaciales, es decir, 2130 casos.

En cuanto al sexo de la muestra, apreciamos que existe mayores fracturas en los hombres $(75.6 \%)$ con relación 3.1:1 siendo un poco más homogéneo en los menores de 14 años (64.7\% para los hombres); este hallazgo coincide con los obtenidos por Erol et al. (2004), quienes encontraron que el $75 \%$ de las lesiones con fractura maxilofacial en una experiencia de 25 años eran hombres. En el estudio de Gomes et al. (2006) de 5 años de seguimiento, el 82.8\% de los casos con fracturas de cigomático fueron hombres, mientras que Chrcanovic et al. (2004) encontraron una relación hombre:mujer de 4.7:1, que es mayor a nuestros resultados. Por otra parte, en un estudio realizado en Irán (Hosein, 2004), la relación hombre mujer fue 3.84:1 lo cual se asemeja un poco más a nuestros resultados. En este sentido, Shaikh \& Worrall (2002) presentaron una investigación con interesantes resultados; ellos estudiaron la epidemiología de las fracturas en población menor a 18 años. En el grupo de 0 a 10 años existió una relación hombre:mujer de 1.5:1, mientras que en el grupo de 11 a 18 años la relación aumento drásticamente hasta 7.6:1. Con esto se confirman nuestros resultados en que a medida que aumentan los años, son más los hombres sujetos a fracturas maxilofaciales.

En cuanto a los grupos etáreos, la mayoría se situó entre los 0 y 19 años (57\%), donde el subgrupo de 0 y 9 años (29\%) fue el de mayor volumen; sobre los 65 años solo un $4.4 \%$ presento fracturas maxilofaciales. En el estudio de Chrcanovic realizado en Brasil, el $43.7 \%$ de las fracturas maxilofaciales se agrupo entre los 20 y 39 años lo cual es levemente mayor a nuestros hallazgos que obtuvieron para este grupo un 37.8\%. Por otra parte, en el trabajo de Gassner et al. (2003), el 50\% tenia entre 10 y 37, lo cual es un poco menor a nuestros resultados (56\% entre 10 y 39 años). Diferentes cifras entrega la investigación de Adebayo et al. (2003), quienes señalan que el $69 \%$ de la demanda por fractura maxilofacial tenía entre 20 y 39 años, lo que es casi el doble de nuestro estudio. Creemos que las diferencias vistas en este análisis podrían deberse a las composiciones poblacionales distintas de algunas investigaciones así como también a las diversas condiciones de vida de tales poblaciones, permitiendo observar pacientes con fracturas maxilofaciales en diferentes condiciones y, por supuesto, en diferentes edades.

Muchos estudios han evaluado la etiología de las fracturas maxilofaciales (Shaikh \& Worrall; Ashraf \& Amin 2005; Iida \& Matsuya 2002; Hosein; Ogundare et al. 2003; Adebayo); estos se han desarrollado en diferentes regiones $\mathrm{y}$ en diferentes edades. En nuestro estudio, la principal causa de fracturas fueron las agresiones (31\%) seguido de cerca por las caídas (24\%); luego, el 11\% sufrió accidentes de tránsito y cerca del $9 \%$ accidentes escolares mientras que el $7 \%$ presentó lesiones por deporte. En este punto de nuestro estudio encontramos un déficit muy importante en el diagnóstico precoz de las fracturas maxilofaciales, ya que en el $17 \%$ de los casos ingresados en el SUHR no se constató la etiología de la fractura, determinando con ello que al cirujano maxilofacial le faltaran herramientas para resolver de mejor forma la situación clínica. En nuestra opinión, el hecho de la ausencia del cirujano oral y maxilofacial en el equipo de urgencia gatillaría en parte esta falencia. 
Estamos de acuerdo con lo señalado por Ogundare et al., quienes plantearon que en la etiología de las fractura maxilofaciales la composición poblacional juegan un rol importante. En el estudio de esos autores, se señala que el $79 \%$ de las fracturas mandibulares se producen por agresiones, mientras que los accidentes de tránsito y las lesiones por armas de fuego alcanzaron un 6\% para cada una; Ogundare et al. también plantean que en su población de estudio (Columbia, USA) existe un nivel socioeconómico medio bajo, crecimiento poblacional en aumento, relativo alto nivel de desempleo y abuso de sustancias tóxicas. Podemos decir que nuestra población tiene un fuerte componente indígena, con más del 50\% de las personas habitando zonas rurales; además, la IX Región de Chile posee las ciudades con los mayores indicadores de alcoholismo del país, mientras que el desempleo rodea el 9\% (Instituto Nacional de Estadística, 2003).

En nuestra población mayor a 15 años, el $46 \%$ presentó fracturas por agresiones y el 14\% accidentes de tránsito, mientras que en un $25 \%$ de las consultas no se consignó la etiología. Podemos presumir que las agresiones se pudieron ver asociadas al fuerte consumo de bebidas alcohólicas y accidentes ocasionadas por ella. Nuestros pacientes con accidentes de tránsito fueron menores a los obtenidos por Hosein y Ashraf \& Amin quienes rodeaban el 55\%. Por otro lado, una investigación que abordaba las fracturas del complejo cigomático, Gomes et al. encontraron mayor presencia de caídas $(21 \%)$ y asaltos $(19.4 \%)$.

En cuanto a la población infantil el 53\% se asoció a las caídas y el $23 \%$ a los accidentes escolares. En la tipificación legal en Chile, los accidentes escolares tienen un "seguro escolar", de modo que el ingreso al SUHR debe registrarse de esa forma; sin embargo, la etiología exacta no se verifica, obstaculizando el proceder del cirujano maxilofacial. Iida \& Matsuya evaluaron 174 niños menores de 16 años, entre los cuales el $51 \%$ presentó fracturas por caídas, lo cual se asemeja a nuestros resultados; otro estudio señala que el $41 \%$ de las fracturas de menores de 11 años son las caídas, junto a un $20 \%$ que presento fracturas asociadas al juego y el deporte (Oji, 1998).

Thomson et al. (2003) en un estudio retrospectivo de 10 años concluyeron que el $71 \%$ de las fracturas maxilofaciales en mayores de 60 años fue por caídas, mientras que Gerbino et al. (1999) encontraron que el 56\% de las fracturas en pacientes sobre 60 años se asocio a las caídas. En nuestro estudio solo el $4.4 \%$ de los fracturados maxilofaciales correspondió a sujetos mayores de 65 años. En cuanto a las fracturas maxilofaciales asociadas al deporte Mourouzis \& Koumoura (2005) lo relacionaron al 6\% de su muestra, lo cual se asemeja al $6.7 \%$ obtenido en nuestro estudio.
Las estructuras comprometidas en las fracturas son variables. En nuestro estudio, las principales estructuras dañada fueron la zona órbito-naso-etmoidal (ONE) en un 56\%, le siguieron las fracturas dentoalveolares en un $22 \%$, las fracturas de mandíbula en un $13 \%$ y las fracturas de hueso cigomático y hueso frontal en $4 \%$ para cada una. Las fracturas de mandíbula en nuestra población son bastante menores a las encontradas en otros estudios; por ejemplo, en el estudio de Ashraf \& Amin, la fractura de mandíbula fue la más común $(51 \%)$ seguida de la fractura de hueso cigomático (17\%). También Chrcanovic señala que la estructura mas dañada en su población es la mandíbula (40\%) seguida de la fractura del hueso cigomático (21\%). Erol et al. también encontraron que la mandíbula fue la más afectada (72.8\%).

En nuestro grupo menor de 15 años, el patrón de fracturas afecto principalmente la zona ONE con un $47.1 \%$ al igual que las fracturas dentoalveolares con un $47.1 \%$; Iida $\&$ Matsuya en sus resultados destacan que el $31 \%$ de los pacientes menores de 16 años tuvo fractura dentoalveolar, mientras solo un $5.3 \%$ presentó fractura de mandíbula. Al contrario de nuestros resultados, Oji señaló que el 89\% de los pacientes menores de 11 años presentaba fracturas de mandíbula.

En el análisis estadístico con Chi-Cuadrado se realizó asociando todas las variables de nuestro estudio. Se obtuvo resultados positivos en solo una pequeña parte de estas. Esto sucedió probablemente por el tamaño muestral reducido de nuestro estudio. Por ejemplo, solo ocurrieron 2 fracturas de hueso frontal y ambos fueron hombres revelando significancia estadística positiva; esto, a nuestro modo de ver, no puede ser clínicamente significativo. Otro punto es que la edad se correlacionó con los accidentes escolares, lo cual podría ser obvio toda vez que en la mayoría de los casos solo los menores de 15 años pueden sufrir este tipo de accidentes.

Sin embargo, la relación dada entre la edad, sexo y agresiones es un muy buen dato, junto a la relación de edad y lesiones dentoalveolares. También se demuestra que las agresiones se relacionan positivamente con fracturas de mandíbula y los accidentes escolares con fracturas dentoalveolares.

Finalmente, podemos concluir que existe un alto número de pacientes con fracturas maxilofaciales de diferente magnitud y compromiso. Esto, junto a los distintos errores en la recepción y transmisión de la información y la ausencia de especialistas para la intervención y diagnóstico precoz justificaría plenamente la presencia de cirujanos y traumatólogos orales y maxilofaciales en los equipos de respuesta inmediata. 
MEDINA, M. J.; MOLINA, P.; BOBADILLA, L. ZAROR, R. \& OLATE, S. Maxillofacial fractures in chilean subjects. Int. J. Morphol., 24(3):423428,2006

SUMMARY: The oral and maxillofacial trauma represent a $6 \%$ to $8 \%$ approximately of the body fractures. These injuries are of complex handling and demand an accurate and precise diagnosis. The Hernán Henríquez Aravena Regional Hospital, of Temuco city, does not have oral and maxillofacial surgeons in the immediate urgency staff, so that first approach to handle those patients is made by other clinicians. After one year, a total of 34,931 consultations were made in the Urgency Service at the Hernán Henríquez Aravena Hospital. Of them, a representative sample of 742 subjects was chosen from which general information and the kind of injuries was obtained. Statistical association with the Chi - Square test was obtained. Six percent of the consultations in the Hernán Henríquez Aravena Regional Hospital were associated to maxillofacial fractures. $64.4 \%$ of the sample corresponded to male subjects. The relation man to woman was 3.1:1, displaying a greater difference in patients aged over 15 years. Of the total of sample, the group with more consultations was those aged below 20 years and the $31,1 \%$ of the consultations were caused by aggressions, whereas $24,4 \%$ were associated to falls. The main zone of fracture was related to the orbit nose etmoidal complex with 55,6\% and dental fractures with a $22.2 \%$. Statistically significance association was found between age, sex, aggression, mandible and dental fractures $(\mathrm{p}<0,05)$ The data reflect the high prevalence of maxillofacial fractures in Temuco, Chile, similar to other parts of the world. The group of young people is the one of main consultation where the aggressions play a fundamental roll. With the present descriptive study we think that the presence of oral and maxillofacial surgeons in the urgency staff of the Hernán Henríquez Aravena Regional Hospital, Temuco, Chile is justified totally.

KEY WORDS: Maxillofacial fractures; Maxillofacial trauma; Epidemiology.

\section{REFERENCIAS BIBLIOGRÁFICAS}

Adebayo, E. T.; Ajike, O. S. \& Adekeye EO. Analysis of the pattern of maxillofacial fractures in Kaduna, Nigeria. $\mathrm{Br}$. J. Oral Maxillofac. Surg., 41:396-400, 2003.

Ashraf, S. \& Amin, F.: Incidence and causes of maxillofacial skeletal injuries at the Mayo Hospital in Lahore, Pakistan. Br. J. Oral Maxillofac. Surg., 43:1-3, 2005.

Bater, M. C. ; Ramchandani, P. L. \& Brennan, P. A. Post-traumatic eye observation. Br. J. Oral Maxillofac. Surg.,43:410-6, 2005.

Chrcanovic, B. R.; Freire-Maia, B.; Souza, N. L.; Araújo, V. O. \& Abreu, M. H. N. G. Facial fractures: a 1-year retrospective study in a hospital in Belo Horizonte. Braz. Oral Res., 18(4):322-8, 2004.

Cunningham, L. \& Hang, R. Management of maxillary fractures. In: Miloro, M.; Ghali, G. E.; Larsen, P. \& Waite, P.; eds. Peterson's principles of oral and maxillofacial surgery. $2^{\circ}$ ed. BC Decker Inc. 2004, p 435-43.

Ellis, E. Soft tissue and dentoalveolar injuries. In: Peterson, L.; Ellis, E.; Hupp, J. \& Tucker, M.; eds. Contemporary Oral and Maxillofacial Surgery. $4^{\circ}$ ed. Mosby, 2003. pp. 504-26.

Erol, B.; Tanrikulu, R. \& Görgün, B. Maxillofacial fractures: analysis of demographic distribution and treatment in 2901 patients (25-year experience). J. CaranioMaxillofac. Surg., 32:308-13, 2004.

Gassner, R.; Tuli, T.; Hächl, O.; Rudisch, A. \& Ulmer, H. Craniomaxillofacial trauma: a 10 year of 9543 cases with 21067 injuries. J. CranioMaxillofac. Surg., 31:51-61, 2003.

Gerbino, G.; Roccia, F.; De Gioanni, P. \& Berrone, S. Maxillofacial Trauma in the elderly. J. Oral Maxillofac. Surg., 57:777-82, 1999.

Gerbino, G.; Roccia, F.; Vence, A. \& Caldarelli, C. Analysis of 158 frontal sinus fractures: current surgical management and complications. J. CranioMaxillofac. Surg., 28:133-9, 2000.
Gomes, P.; Passeri, L. \& Albergaría Barbosa, J. A 5-year restrospective study of zigomatico-orbital complex and zygomatic arch fractures in Sao Paulo State, Brazil. J. Oral Maxillofac. Surg., 64:63-7, 2006.

Hosein, M. Maxillofacial fractures in Hamedan province, Iran: a retrospective study (1987-2001). J. CranioMaxillofac. Surg., 32: 28-34, 2004.

Iida, S. \& Matsuya, T. Paediatric maxillofacial fractures: their aetiological characters and freature patterns. $J$. CranioMaxillofac. Surg., 30:237-41, 2002.

Instituto Nacional de Estadísticas. Censo 2002, síntesis de resultados. Santiago de Chile, 2003.

Mourouzis, C. \& Koumoura, F. Sports - related maxillofacial frectures : a retrospective study of 125 patients. Int. J. Oral Maxillofac. Surg., 34:635-8, 2005.

Ogundare, B.; Bonnick, A. \& Bayley, N. Pattern of mandibular fractures in a urban major trauma center. J. Oral Maxillofac. Surg., 61:713-8, 2003.

Oji, Ch. Fractures of the facial skeleton in children: a survey of patients under the age of 11 years. J. CranioMaxilofac. Surg., 26: 322-5, 1998.

Shaikh, Z. \& Worrall, S. Epidemiology of facial trauma in a simple of patients aged $1-18$ years. Int. J. Care Injured, 33:669-71, 2002.

Thomson, M.; Stephenson, S.; Kieser, J. A. \& Lagley, J. D. Dental and maxillofacial injuries among older New Zealanders during the 1990s. Int. J. Oral Maxillofac. Surg., 32:201-5, 2003.

Dirección para correspondencia:

Dr. Sergio Olate Morales

Universidad de La Frontera,

Facultad de Medicina

Departamento de Odontologia Integral

Manuel Montt 112, Piso 4․

Temuco, CHILE

Email:solate@ufro.cl

Recibido : 23-03-2006

Aceptado: 28-06-2006 